\title{
LOS ISÓTOPOS ESTABLES DEL HIDRÓGENO Y EL OXÍGENO EN LOS ESTUDIOS ECOFISIOLÓGICOS DE PLANTAS
}

\author{
CASANDRA ReYes-García y JOSÉ LUIS ANDRADE \\ Unidad de Recursos Naturales, Centro de Investigación Científica de Yucatán, A. C., Calle 43 No. 130. \\ Colonia Chuburná de Hidalgo, C. P. 97200, Mérida, Yucatán, México. \\ 'Autor para correspondencia. Tel. 52 (999) 9813914 ext. 369. Fax: 52 (999) 9813900. \\ Correo-e: andrade@cicy.mx
}

\begin{abstract}
Resumen: Los estudios con los isótopos estables de elementos de interés biológico se han convertido en una herramienta útil para conocer el intercambio de las moléculas en la biosfera. Como el agua es una de las moléculas más abundantes en dicho intercambio, los estudios con los isótopos estables del hidrógeno y el oxígeno han sido un componente fundamental de muchos trabajos ecofisiológicos con plantas, desde el nivel de hoja hasta la reconstrucción de climas pasados. En esta revisión mencionamos la metodología más utilizada, la notación general y las investigaciones más relevantes con estos isótopos. Asimismo, revisamos estudios sobre las fuentes de agua de las plantas, el enriquecimiento isotópico en las hojas debido a la transpiración, la relación del ambiente con los isótopos del oxígeno en la materia orgánica, así como algunos estudios recientes que nos han permitido proponer a algunas especies como indicadoras ambientales en un mundo con cambio global.
\end{abstract}

Palabras clave: firmas isotópicas, fraccionamiento, fuentes de agua, indicadores ambientales.

\begin{abstract}
Stable isotope studies of elements in biological organisms have become a useful tool to assess the exchange of molecules in the biosphere. Since water is one of the most abundant molecules in such an exchange, studies on stable isotopes of hydrogen and oxygen have become a fundamental component of many plant ecophysiological studies, from the leaf level to the reconstruction of past climates. In this review, we mention the most common methodologies, general notation and the most relevant research on hydrogen and oxygen stable isotopes. Also, we discuss studies on plant water sources, leaf isotopic enrichment due to transpiration, the relationship between environment and oxygen stable isotopes in organic matter, and present studies that propose some plant species as environmental indicators in a globally changing world.
\end{abstract}

Key words: environmental indicators, fractionation, isotopic signatures, water sources.

$\mathbf{M}$ uchos elementos químicos de interés biológico poseen dos o más formas, con el mismo número atómico pero con diferente masa (isótopos estables). Es decir, los isótopos estables tienen el mismo número de protones y de electrones, pero diferente número de neutrones. En general, los isótopos estables de un elemento se encuentran en diferente abundancia: uno de los isótopos está en mayor abundancia y uno o dos isótopos en menor abundancia (cuadro 1). Debido a que el comportamiento físico y químico es diferente para cada uno de los isótopos estables de un elemento, éstos pueden usarse como trazadores de moléculas en la biosfera e incluso en experimentos con compuestos enriquecidos en alguno de los isótopos, con el fin de evaluar el movimiento de los recursos de plantas y animales a través de los ecosistemas (Dawson et al., 2002; Santiago et al., 2005).

En esta revisión trataremos solamente los isótopos estables más abundantes del hidrógeno $\left[{ }^{1} \mathrm{H},{ }^{2} \mathrm{H}\right.$ o deuterio (D)] y del oxígeno $\left({ }^{16} \mathrm{O},{ }^{18} \mathrm{O}\right)$, enfocándonos en la molécula de agua $\left(\mathrm{H}_{2} \mathrm{O}\right)$ debido a que es la forma más abundante de intercambio entre el ambiente y los organismos. Discutirmos la metodología y los estudios que se han realizado principalmente en los ecosistemas tropicales. Asimismo, dirigiremos a los lectores a información más detallada sobre los temas de mayor actualidad y mencio na remos algunas perspectivas de investigación fisiológi ca con esta metodología. 
Cuadro 1. Abundancia promedio en la Tierra de los isótopos estables de elementos de interés biológico (tomado de Ehleringer y Osmond, 1989).

\begin{tabular}{lcc}
\hline Elemento & Isótopo & $\begin{array}{c}\text { Abundancia promedio } \\
\text { en la Tierra (\%) }\end{array}$ \\
\hline Hidrógeno & ${ }^{1} \mathrm{H}$ & 99.985 \\
& ${ }^{2} \mathrm{H}$ o D & 0.015 \\
Carbono & ${ }^{12} \mathrm{C}$ & 98.89 \\
& ${ }^{13} \mathrm{C}$ & 1.11 \\
Oxígeno & ${ }^{16} \mathrm{O}$ & 99.759 \\
& ${ }^{17} \mathrm{O}$ & 0.037 \\
Nitrógeno & ${ }^{18} \mathrm{O}$ & 0.204 \\
& & 99.63 \\
Azufre & ${ }^{14} \mathrm{~N}$ & 0.37 \\
& ${ }^{15} \mathrm{~N}$ & 95.0 \\
& ${ }^{32} \mathrm{~S}$ & 0.76 \\
& ${ }^{33} \mathrm{~S}$ & 4.22 \\
& ${ }^{34} \mathrm{~S}$ & 0.014 \\
\hline
\end{tabular}

\section{Notación y metodología}

Debido a que la variación absoluta en la abundancia natural de la mayoría de los isótopos es muy baja, convencionalmente se reportan las variaciones relativas de los isótopos con respecto a un estándar internacional. Para el caso del $\mathrm{H}$ y del O, el estándar es el agua oceánica VSMOW (Viena-Standard Mean Ocean Water), cuya abundancia isotópica absoluta del hidrógeno es 99.984426 átomo por ciento de ${ }^{1} \mathrm{H}$ y 0.015574 átomo por ciento de D (Hagemann et al., 1970); la abundancia isotópica absoluta del oxígeno en VSMOW es 99.76206 átomo por ciento de ${ }^{16} \mathrm{O}, 0.03790$ átomo por ciento de ${ }^{17} \mathrm{O}$ y 0.20004 átomo por ciento de ${ }^{18} \mathrm{O}$ (Baertschi, 1976).

La notación resultante es conocida como delta $(\delta)$ en partes por mil (\%o), la cual es calculada como:

$$
\delta=\left(\frac{R_{\text {muestra }}}{R_{\text {estandar }}}\right)-1 \times 1000
$$

Donde $R$ indica el cociente del isótopo pesado sobre el isótopo liviano, $R_{\text {muestra }}$ y $R_{\text {estandar }}$ se refieren a la abundancia de $\mathrm{D} / \mathrm{H}\left(\mathrm{u}{ }^{18} \mathrm{O} /{ }^{16} \mathrm{O}\right)$ en la muestra y en el estándar, respectiva-

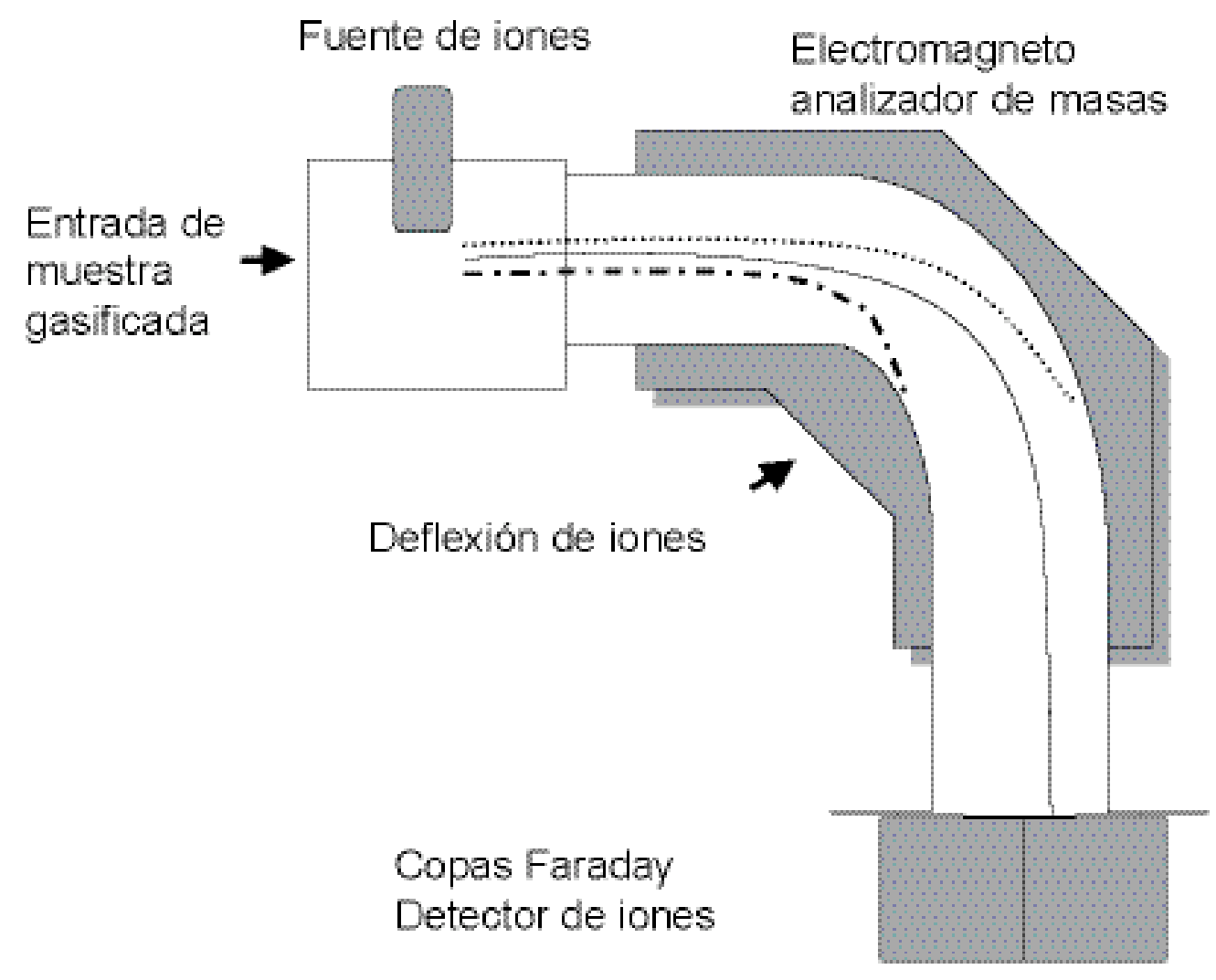

Figura 1. Esquema de un espectrómetro de masas. La muestra ingresa en forma de gas por el sistema y es ionizada en la fuente de iones. Al pasar por el electromagneto, los iones sufren una deflexión correspondiente a su carga y masa (como ejemplo se muestra la trayectoria de tres iones representados por tres líneas: una sólida, una punteada y una de puntos y rayas). Los iones recolectados en las copas Faraday son amplificados y su concentración se determina al compararse con un estándar. 
mente. Un valor $\delta$ más positivo indica que la muestra tiene una mayor proporción del isótopo pesado en comparación con el estándar.

Para medir los isótopos estables se usa el espectrómetro de masas, siguiendo una metodología prácticamente no destructiva, ya que una pequeña muestra de tejido (albura, raíz u hoja) es suficiente para el análisis. El espectrómetro de masas separa átomos y moléculas cargadas con base en su movimiento en campos magnéticos o eléctricos y consiste de cuatro partes centrales (Hoefs, 2004): el sistema de entrada, la fuente de iones, el analizador de masas y el detector de iones (figura 1).

El sistema de entrada requiere de un alto vacío y los compuestos son introducidos al sistema como gases; en este sistema se utiliza un flujo de gases viscoso para evitar la discriminación de masas. En la fuente de iones, se forman los iones, se aceleran y se enfocan en un haz estrecho; generalmente, los iones son producidos por bomb a rdeo de electrones y los iones positivos que se forman y entran al campo magnético poseen la misma energía cinética. El analizador de masas separa los haces de iones que provienen de la fuente de iones de acuerdo con los cocientes de masa/carga (m/q) mediante un campo magnético; éstos son desviados en vías circulares, cuyos radios son proporcionales a la raíz cuadrada de m/q. Después de pasar por el campo magnético, los iones separados son recolectados en detectores de iones (copas Fa rad ay). Las copas Fa rad ay poseen un resistor y el paso de los iones a través de estos detectores crea una caída de potencial en el resistor que actúa como una medida de la comiente de los iones (Ehleringer y Osmond, 1989; Hoefs, 2004).

\section{Firmas isotópicas, fraccionamiento y enriquecimiento}

$\mathrm{Al}$ valor $\delta$ de una sustancia (por ejemplo, agua de una hoja, lluvia, celulosa) se le denomina firma isotópica de dicha sustancia. Debido a que los neutrones ejercen una influencia sobre la masa atómica, los isótopos pesados y ligeros se comportan de manera diferente durante las reacciones físicas y químicas. Cuando esto ocurre, las firmas isotópicas de las sustancias cambian y a este proceso se le denomina fraccionamiento. Por ejemplo, los diferentes tipos de agua (subterránea, pluvial, edáfica, fluvial u oceánica) tienen composiciones isotópicas diferentes debido a los procesos de evaporación y precipitación. En consecuencia, las composiciones isotópicas de los diferentes componentes del ciclo del agua muestran firmas isotópicas bien definidas, las cuales pueden ser identificadas y así describirse sus interrelaciones.

Cuando se presenta un fraccionamiento de una fuente de agua, por ejemplo, como producto de la evaporación, se puede presentar la composición isotópica como un enriquecimiento (un incremento en el isótopo más pesado) sobre la fuente de agua inicial, la cual puede calcularse como (por ejemplo, para el caso del ${ }^{18} \mathrm{O}$ ):

$$
\Delta{ }^{18} O_{\text {producto }}=\frac{R_{\text {producto }}}{R_{\text {fuente }}}-1
$$

Donde $\mathrm{R}_{\text {producto }}$ es el cociente ${ }^{18} \mathrm{O} /{ }^{16} \mathrm{O}$ del producto y $\mathrm{R}_{\text {fuente }}$ es el cociente ${ }^{18} \mathrm{O} /{ }^{16} \mathrm{O}$ de la fuente de agua. Como aproximación se puede también estimar como:

$$
\Delta{ }^{18} O_{\text {producto }}=\delta^{18} O_{\text {producto }}-\delta^{18} O_{\text {fuente }}
$$

\section{Fuentes de agua}

En las fuentes de agua dulce del planeta se ha encontrado una correlación entre $\delta \mathrm{D}$ y $\delta^{18} \mathrm{O}$ que se conoce como línea meteórica (Gat, 1996; figura 2). Esta línea meteórica muestra una variación lineal entre ambos isótopos que permite utilizarlos de manera intercambiable en estudios de absorción diferencial de agua, así como combinar estudios de abundancia natural con enriquecimiento en alguno de los dos isótopos pesados derivado de procesos ecofisiológicos (Dawson et al., 2002).

Los isótopos estables del hidrógeno y los del oxígeno pueden usarse para determinar la procedencia del agua contenida en el xilema de los árboles porque durante el proceso de absorción radicular no ocurre fraccionamiento isotópico (White et al., 1985). Entonces, la composición isotópica del agua del xilema refleja las fuentes de agua empleadas por las raíces (Meinzer et al., 2001; Goldstein et al., 2002; Andrade, 2005; Santiago et al., 2005), con la posible excepción de los mangles (Sternberg y Swart, 1987). Con el uso de las firmas de $\delta \mathrm{D}$ se ha determinado

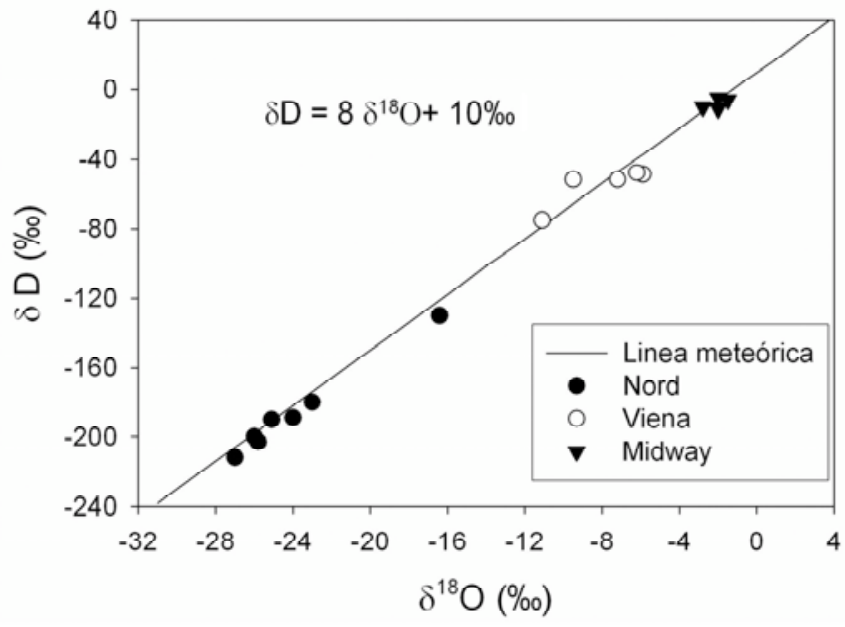

Figura 2. Línea meteórica que muestra la relación de $\delta^{18} \mathrm{O}$ y $\delta \mathrm{D}$ en varias estaciones de colecta. Las estaciones en la parte inferior izquierda de la línea se encuentran en latitudes más altas, y presentan temperaturas medias menores, lo cual deriva en lluvia con valores más negativos de $\delta^{18} \mathrm{O}$ y $\delta \mathrm{D}$; las estaciones de la parte superior derecha se acercan al ecuador (adaptado de Gat et al., 2000). 
que una hemiepifita depende sólo del agua de la niebla durante sus primeros estadios de vida, y que cambia progresivamente hacia agua de lluvia en cuanto sus raíces llegan al suelo del bosque (Feild y Dawson, 1998).

En varios ecosistemas, el enriquecimiento isotópico de los horizontes superiores del suelo ocurre porque la evaporación causa que el agua de los horizontes más superficiales se enriquezca con los isótopos más pesados. Por esta razón, después de un período largo sin lluvias se forma un perfil, con un gradiente de agua progresivamente empobrecida en $\delta \mathrm{D}\left(\mathrm{o} \delta^{18} \mathrm{O}\right)$ desde la superficie hasta los horizontes más profundos (figura 3), con el que se pueden comparar los valores de $\delta \mathrm{D}$ o $\delta^{18} \mathrm{O}$ del agua del xilema de las especies leñosas (Meinzer et al., 2001; Goldstein et al., 2002; Andrade, 2005; Santiago et al., 2005).

Con esta metodología se puede conocer la partición del agua entre diferentes especies en una comunidad (Ehleringer et al., 1991; Jackson et al., 1995; Phillips y Ehleringer, 1995; Jackson et al., 1999). Entre los árboles perennifolios tropicales, los individuos con diámetros menores absorben el agua preferentemente de los horizontes más profundos que los árboles de diámetros mayo re s (Meinzer et al., 1999) y las lianas acceden al agua de capas más profundas de suelo que los árboles, pero aquéllas también utilizan el agua de horizontes aún más profundos conforme avanza la estación seca (Andrade et al., 2005).

Va rios estudios han usado fuentes de agua enriquecida en algún isótopo estable para ras trear el movimiento del agua en el suelo. De esta manera se ha demostrado la redistribu- ción hidráulica en suelos tropicales (Moreira et al., 2003) y la extensión de las raíces laterales de algunos árboles (Stemberg et al., 2002). También, con el estudio de los isótopos estables de $\mathrm{H}$ y $\mathrm{O}$ se ha detectado el efecto de los cambios ambientales en los ciclos hidrológicos regionales y globales (Moreira et al., 1997; Henderson-Sellers et al., 2002; Martinelli et al., 2004), así como en los procesos fisicoquímicos y bioquímicos a nivel de hoja y otros órganos de la planta (Cernusak et al., 2003; Stemberg et al., 2003).

\section{Transpiración y enriquecimiento foliar en $\delta^{18} \mathrm{O}$}

Durante la evaporación los isótopos más ligeros tienden a evaporarse primero, por lo que el vapor presenta un valor empobrecido en ${ }^{18} \mathrm{O}$, con un valor de $-9 \%$ con respecto al agua fuente (dictado por un factor de fraccionamiento de 1.0098 a $20^{\circ} \mathrm{C}$; Majoube, 1971; Gat, 1996); lo contrario sucede durante la condensación. Si el sistema en el que ocurre este fraccionamiento es abierto y el vapor de agua es removido inmediatamente después de la evaporación, el enriquecimiento en el isótopo más pesado del cuerpo de agua continuaría hasta que todos los isótopos ligeros se evaporaran (Gat, 1996). Sin embargo, en un sistema natural, las fases líquidas y gaseosas están en un equilibrio dinámico, y se componen de dos flujos, el agua que se evapora y el vapor que se condensa. Por esta razón, en condiciones de humedad y temperatura constantes, los cuerpos de agua llegan a un estado isotópico estable, en el cual ya no se da más enriquecimiento del cuerpo de agua

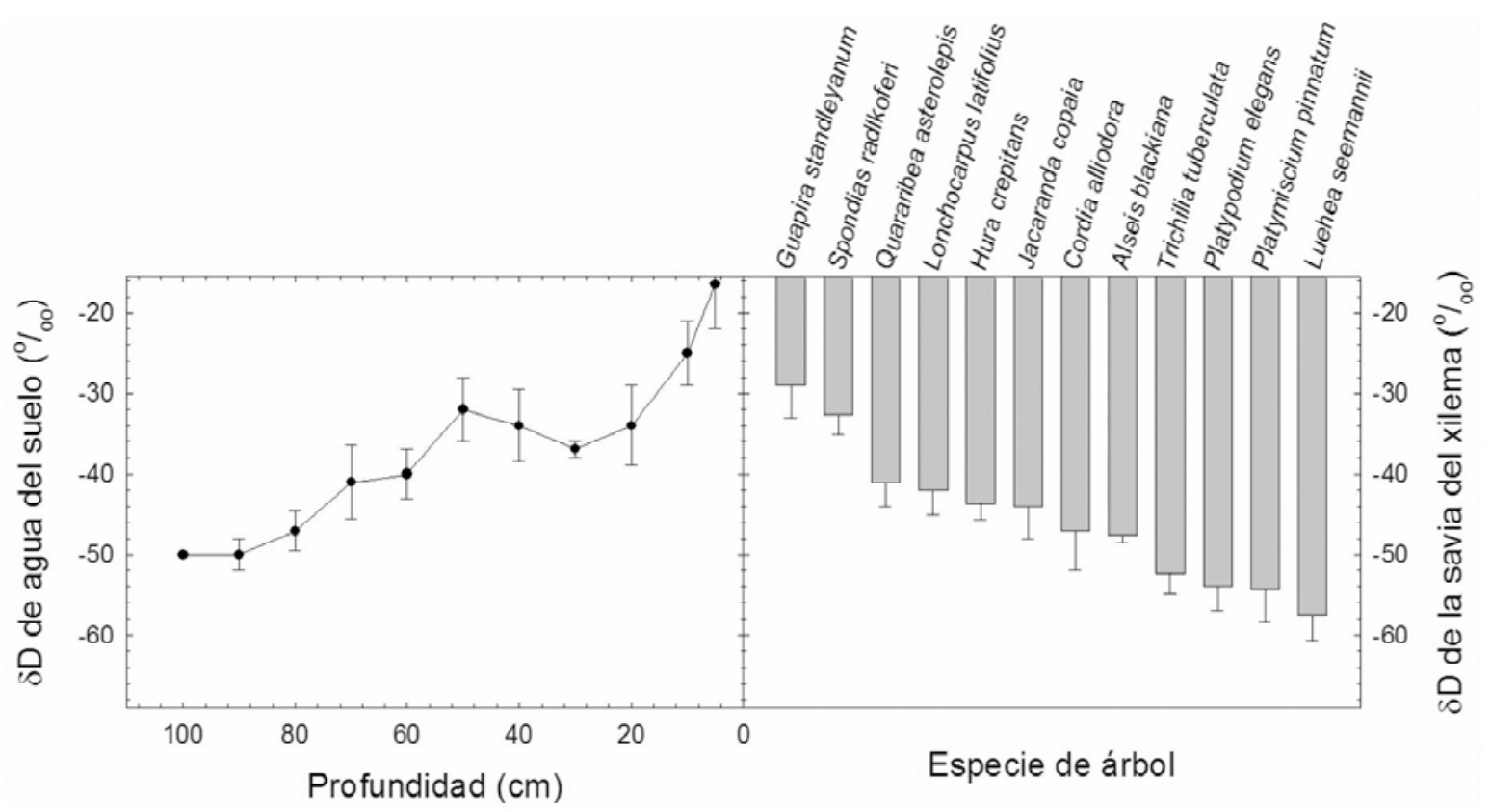

Figura 3. Contenido isotópico $(\delta \mathrm{D})$ del agua del suelo con relación a la profundidad del suelo (panel izquierdo) y $\delta \mathrm{D}$ del agua de los vasos del xilema de 12 especies de árboles en un bosque de Panamá (panel derecho). La toma de muestras se realizó después de 40 días de sequía (adaptado de Meinzer et al., 1999). 
líquida y el vapor permanece con una firma isotópica estable (Gat, 1996). La ecuación de Craig-Gordon (Craig y Gordon, 1965) describe la relación entre la temperatura, la humedad, y ambos factores de fraccionamiento por cambio de fase y difusión, lo que logra predecir el enriquecimiento de un cuerpo de agua en diferentes condiciones de evaporación. La señal isotópica del cuerpo de agua en evaporación es directamente proporcional a la temperatura e inversamente proporcional a la humedad relativa, esto es, mayores temperaturas y una humedad relativa baja resultarán en menor enriquecimiento del cuerpo de agua.

Cuando el agua se evapora de la cavidad subestomática en las hojas, se lleva a cabo un proceso similar al descrito para un cuerpo de agua y el agua de la hoja queda enriquecida en los isótopos pesados (figura 4). En condiciones de humedad y temperatura constantes, el enriquecimiento en $\delta^{18} \mathrm{O}$ continúa hasta que se alcanza un estado isotópico estable, donde la señal del agua de la hoja no cambia (Flanagan et al., 1991). Sin embargo, en un ambiente natural, las variaciones constantes en radiación conducen a condiciones de temperatura y humedad cambiantes, y es frecuente que el estado isotópico estable no se alcance (Harwood et al., 1998). El tiempo de recuperación del estado estable varía de especie a especie, y para plantas de cultivo es de 2-3 h (Bariac et al., 1989; Flanagan et al., 1991; Yakir et al., 1994), pero esto depende principalmente del tiempo de residencia del agua foliar (Farris y Strain, 1978; Allison et al., 1985; Cooper y DeNiro, 1989; Tissue et al., 1991; Wang y Yakir, 1995; Harwood et al., 1998; Harwood et al., 1999; Pendall et al., 2005). Este índice representa el tiempo que toma el agua foliar en ser completamente reemplazada y depende de la tasa de transpiración y del volumen de agua foliar. En las plantas suculentas el estado estable isotópico sería difícil de alcanzar por la gran cantidad de agua foliar y las bajas tasas de transpiración.

\section{Firmas de $\delta^{18} O$ dentro de las hojas}

Las venas foliares, las cuales reciben agua con la misma firma isotópica que las raíces, están constantemente diluyendo el agua fraccionada en las cavidades subestomáticas (Farquhar y Lloyd, 1993; Farquhar y Gan,

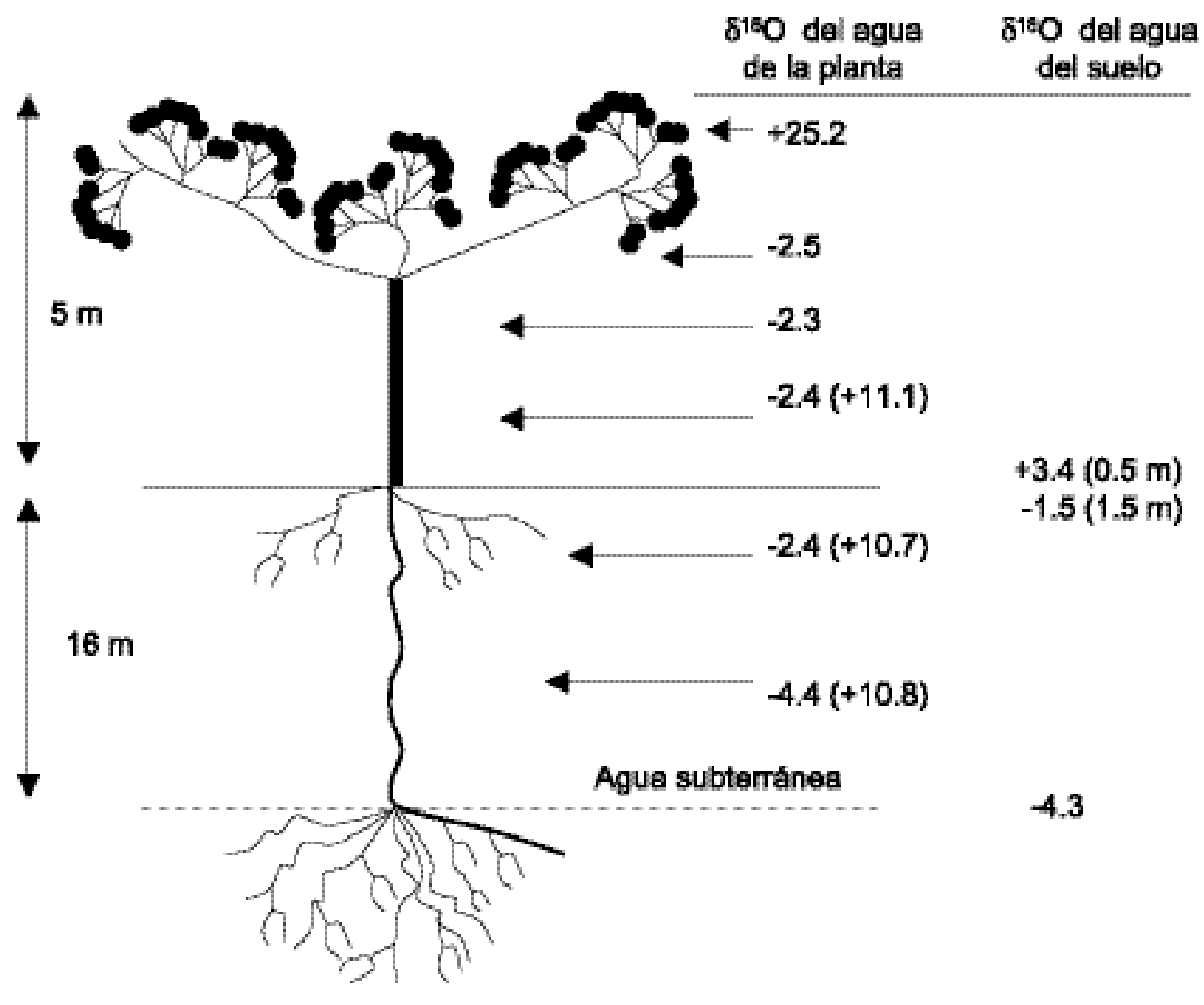

Figura 4. Variaciones en $\delta^{18} \mathrm{O}$ del agua del árbol Tamarix jordanis y su relación con los valores de $\delta^{18} \mathrm{O}$ del agua del suelo y subsuelo. El agua de las muestras de tallo y raíces se extrajo del xilema y el floema (los valores del floema se encuentran entre paréntesis). El árbol que presenta raíces superficiales y profundas se encontraba creciendo en una duna en Negev, Israel, y al momento de la colecta presentó firmas isotópicas tanto del agua de estratos superficiales como del agua subterránea. El floema presentó valores muy enriquecidos de $\delta^{18} \mathrm{O}$ (adaptado de Yakir, 1998). 
2003). Debido a esto, existen gradientes de enriquecimiento isotópico dentro de la hoja, desde las células alrededor de las cavidades subestomatales (altamente enriquecidas en isótopos pesados) hasta las células que rodean las venas principales (no fraccionadas isotópicamente), lo que genera una cadena de zonas foliares con valores intermedios de $\delta^{18} \mathrm{O}$. Este fenómeno de formación de un gradiente de $\delta^{18} \mathrm{O}$ en el agua, desde las cavidades subestomatales hasta las venas principales, se conoce como efecto 'Péclet' (Farquhar y Lloyd, 1993). El efecto Péclet predice una respuesta reducida del fraccionamiento del agua a los cambios en la humedad relativa del ambiente, pero una respuesta notable ante los cambios en la conductancia estomática (Barbour et al., 2005). Barbour et al. (2000b) usaron el modelo de Craig-Gordon para describir la señal isotópica de la sacarosa, la cual refleja la señal del agua de la hoja, y demostraron que se obtienen valores teóricos más sensibles a cambios en la tasa de transpiración que los valores reales y además, al tomar en cuenta el efecto Péclet, esta diferencia disminuye.

Aunque el efecto Péclet observado alrededor de las venas reticulares de las dicotiledóneas es pequeño, en las monocotiledóneas se presentan grandes diferencias entre la base y el ápice, especialmente en las que tienen hojas largas con venación paralela (Helliker y Ehleringer, 2000). En estas últimas plantas, la señal es progresivamente enriquecida desde la parte proximal a la parte distal de la hoja debido al enriquecimiento del agua de la vena durante su recorrido hacia el ápice (Helliker y Ehleringer, 2000, 2002a, b; Barnes et al., 2004). Asimismo, una reducción en la capa límite en la parte distal de estas hojas acentúa el efecto Péclet porque la conductancia total al agua aumenta en los ápices (Nobel 1991, Andrade, 2003).

Por lo descrito hasta aquí, la señal isotópica del agua de la hoja depende principalmente de la señal isotópica del agua proveniente de la raíz, del grado de evaporación y de la humedad relativa. Estudios recientes han demostrado que la apertura de estomas durante la noche, o en momentos con muy alta humedad relativa, puede llevar a que la señal isotópica de la hoja se vea influenciada por la señal del vapor de agua en la atmósfera (Cernusak et al., 2005; Seibt et al., 2006; Helliker y Griffiths, 2007). Esto es más evidente en plantas con metabolismo ácido de las crasuláceas (CAM por sus siglas en inglés), cuyo intercambio gaseoso se realiza casi exclusivamente por la noche, y cuya señal isotópica puede reflejar al $100 \%$ la del vapor atmosférico (Helliker y Griffiths, 2007).

\section{$\delta^{18} \mathrm{O}$ y materia orgánica}

Las mediciones de $\delta^{18} \mathrm{O}$ en material orgánico vegetal se utilizan para un sinnúmero de aplicaciones. Por ejemplo, en la reconstrucción de paleoclimas se puede conocer la composición isotópica del ox í geno de las lluvias, la temperat u ra y la humedad cientos de años atrás con base en los isótopos de la celulosa en los anillos de crecimiento de los árboles (Burk y Stuiver, 1981; Yakir et al., 1994; Yakir, 1998). Además, el análisis del $\delta^{18} \mathrm{O}$ nos puede revelar el ori gen de ciertos mat e riales (papel, tela, utensilios) y así ser empleado en estudios de dife rente índole, incluyendo los estudios forenses y arqueológicos (Gat, 1998; Pollard 1998). Fnalmente, las mediciones del $\delta^{18} \mathrm{O}$ pueden ayudar a interp retar las dife rencias en la discriminación de los isótopos de carbono entre individuos que crecen en el mismo ambiente, porque la señal isotópica del ${ }^{13} \mathrm{C}$ responde a cambios en la conductividad estomática y en la tasa fotosintética (Fa rquhar et al., 1982; Santiago et al., 2005), mientras que la señal de ${ }^{18} \mathrm{O}$ es independiente de la tasa fotosintética, pero d epende de la conductividad estomática y la fuente de agua (Barbour et al., 2000a). Por lo tanto, mediciones conjuntas de ambos isótopos estables en condiciones similares permiten infe rir la conductancia estomática y la eficiencia del uso de agua de manera rápida y confiable (Fa rquhar et al., 1998; Barbour et al., 2005; Fa rquhar y Cernusak, 2005).

Temperat u ras bajas en el momento de la condensación, mayor altitud, 1 atitud y distancia de la costa resultan en valores bajos de $\delta \mathrm{D}$ y $\delta^{18} \mathrm{O}$ en la precipitación (figura 2; Dansgaard, 1964; Siegenthaler y Oeschger, 1980; Rozanski et al., 1993; Bowen y Wilkinson, 2002). La señal isotópica de la precipitación local es monitoreada hoy en día por diferentes grupos en todo el mundo que colaboran para registrar estos datos en la Global Network of Isotopes in Precipitation, asociación que utiliza las señales isotópicas para evaluar cambios en el ciclo hidrológico y en el clima, que podrían reflejarse en alteraciones progresivas de los valores medios de $\delta \mathrm{D}$ y $\delta^{18} \mathrm{O}$ (IAEA/WMO, 2004).

Además de monitorear cambios en el valor isotópico de la precipitación hoy en día, el monitoreo de $\delta^{18} \mathrm{O}$ en la celulosa en los anillos de crecimiento de los árboles y en los depósitos de este material en los sedimentos lacustres, p e rmiten evaluar cambios en el valor isotópico de la precipitación y la humedad relativa durante la formación de la celulosa (Gray y Thompson, 1976; Epstein et al., 1977; Burk y Stuiver, 1981; Yapp y Epstein, 1982; Yakir y DeNiro, 1990; Aucour et al., 1996; Barbour et al., 2000b). Al formarse la celulosa, sus átomos de oxí geno se intercambian con el agua celular durante la reacción de hidratación de los carbonilos (Sternberg y DeNiro, 1983; Sternberg et al., 1986; Yakir y DeNiro, 1990). Esta reacción deriva en un fraccionamiento de la señal isotópica, dejando a la molécula de celulosa enriquecida en un $27 \%$ o con respecto al agua de la hoja o tallo donde se formó (DeNiro y Epstein, 1981; Sternberg, 1989).

De esta manera puede correlacionarse directamente la señal isotópica de la materia orgánica con la de la lluvia durante su formación, ya que el agua que llega a las hojas de árboles no tiene ningún fraccionamiento isotópico (White et al., 1985). Como alternativa, si por otros medios 
independientes se tiene el valor isotópico de la lluvia, puede deducirse la humedad relativa al momento de la formación de la celulosa (Yakir et al., 1994; Aucour et al., 1996; Farquhar et al., 1998).

\section{Modelos de circulación y productividad global}

Dado que el planeta puede considerarse como un sistema cerrado en cuanto a la circulación de los elementos, los isótopos estables pueden utilizarse como trazadores en la biosfera a través del tiempo. Un ejemplo de esto es el caso del oxígeno libre $\left(\mathrm{O}_{2}\right)$ que se encuentra en la atmósfera, el cual tiene un periodo de residencia de 1,200 años (Bender et al., 1994) y presenta una señal isotópica de $\delta^{18} \mathrm{O}$ de $23.5 \%$ por encima de la señal de $0 \%$ del océano (Dole, 1935). A esta discrepancia se le conoce como efecto Dole, el cual se explica principalmente por la respiración (que resulta en un enriquecimiento de 20\%o) y debido a que el $\mathrm{O}_{2}$ de la atmósfera es influenciado por el $\delta^{18} \mathrm{O}$ del agua de las hojas que, como hemos visto en secciones anteri ores, en plantas temestres tiende a enriquecerse debido a la transpiración (Bender et al., 1994; Beerling, 1999; Hoffmann et al., 2004). Con la precisión de medición actual, no se detectan diferencias en la concentración de isótopos de oxígeno entre los hemisferios norte y sur. Sin embargo, existe una diferencia de hasta $0.7 \%$ con burbujas de aire atrapadas en glaciares polares o en sedimentos marinos, que denotan cambios importantes en los ciclos hídricos y del carbono en el pasado (Bender et al., 1994; Malaize et al., 1999; Hoffmann et al., 2004). Para poder entender las variaciones de productividad entre periodos glaciares e interglaciares se han realizado diferentes estudios para comprender y modelar el enriquecimiento de $\delta^{18} \mathrm{O}$ en el agua de la hoja en los diversos ecosistemas.

Por el momento, uno de los factores de error más grandes en los modelos es la ausencia de mediciones de $\delta^{18} \mathrm{O}$ del vapor de agua en la atmósfera. La señal de vapor de agua es difícil de medir debido a la costosa y complicada metodología (Helliker et al., 2002), por lo cual generalmente se supone que esta señal se encuentra equilibrada con grandes cuerpos de agua cercanos o con la lluvia. Sin embargo, algunos estudios han demostrado que esto no siempre se cumple (Danis et al., 2006), e incluso que la señal puede tener grandes variaciones a lo largo del día (Yakir, 1998; Harwood et al., 1999). La reciente observación de que epifitas con fotosíntesis CAM pueden ser marcadores de la señal isotópica del vapor de agua de la atmósfera abre nuevas posibilidades de investigación y de uso de estas plantas como indicadoras ambientales (Helliker y Griffiths, 2007). Como estas epifitas realizan el intercambio gaseoso bajo condiciones de alta humedad atmosférica y son además independientes del agua del suelo, el intercambio con las moléculas de agua de la atmósfera es muy grande. Esto hace que el agua de sus hojas presente la misma señal que el vapor atmosférico y no la de la lluvia o riego (cuadro 2). La señal del agua de las hojas queda a su vez depositada en la materia orgánica de la hoja. Debido a este proceso, material de herbario de las epifitas recolectadas hace cien años puede darnos valores isotópicos del vapor atmosférico cuando la planta estaba en crecimiento (figura 5).

Contar con valores de $\delta^{18} \mathrm{O}$ del vapor de agua de la atmósfera nos permite a su vez recalibrar los valores de temperatura, humedad y precipitación infe ridos a partir de anillos de crecimiento de los árboles, mencionado en las secciones previas. Dentro de esta metodología de reconstru cción paleoclimática, también los datos de vapor atmosférico son un gran faltante. Dado que la fisiología de la planta (por ejemplo, los controles estomáticos y la estructura del sistema radical) también puede influir sobre el valor isotópico de la materia orgánica en anillos de crecimiento, el contar con más elementos de información externos nos permitirá afinar nuestras predicciones. Actualmente se está trabajando para encontrar epifitas ideales para este tipo de estudios (C. Reyes-García, M. Mejía-Chang y H. Griffiths, datos sin publicar).

\section{Perspectivas}

Los isótopos estables constituyen una herramienta cuya metodología se ha vuelto accesible para los fisiólogos en las últimas tres décadas. Desde entonces diversos trabajos de campo y de laboratorio han permitido el desarrollo de su uso como trazador y en la elaboración de modelos que integran desde procesos fisiológicos en las plantas a nivel celular, hasta los intercambios entre la planta y su medio, recomiendo incluso ciclos globales de productividad marina versus terrestre. Estos estudios han permitido a su vez

Cuadro 2. Valores de $\delta^{18} \mathrm{O}(\%)$ del agua de las hojas de una bromeliácea con fotosíntesis CAM bajo diferentes tratamientos de humedad relativa y fuentes de agua con valores contrastantes de $\delta^{18} \mathrm{O}$. La especie Tillandsia intermedia se mantuvo en un invernadero con temperatura constante $\left(17^{\circ} \mathrm{C}\right)$ y variaciones en humedad relativa $60-100 \%$. La primera columna muestra el valor de $\delta^{18} \mathrm{O}$ del agua de la hoja después de 15 días de crecimiento bajo las condiciones descritas. Cuando la humedad relativa era alta (75-100\%) el agua de la hoja presentó valores similares al valor del vapor atmosférico condensado (Reyes-García y Griffiths, sin publicar).

\begin{tabular}{cccc}
\hline $\begin{array}{c}\text { Agua de la } \\
\text { hoja } \delta^{18} \mathrm{O} \\
(\%)\end{array}$ & $\begin{array}{c}\text { Vapor atmosférico } \\
\text { condensado } \\
\delta^{18} \mathrm{O}(\%)\end{array}$ & $\begin{array}{c}\text { Agua de riego } \\
\delta^{18} \mathrm{O}(\%)\end{array}$ & $\begin{array}{c}\text { Humedad } \\
\text { relativa } \\
(\%)\end{array}$ \\
\hline-8.4 & -9.8 & -12 & 100 \\
-1.7 & 0.3 & -17 & 75 \\
3 & 0.3 & -6 & 75 \\
6.5 & -6.3 & -6 & 60 \\
\hline
\end{tabular}




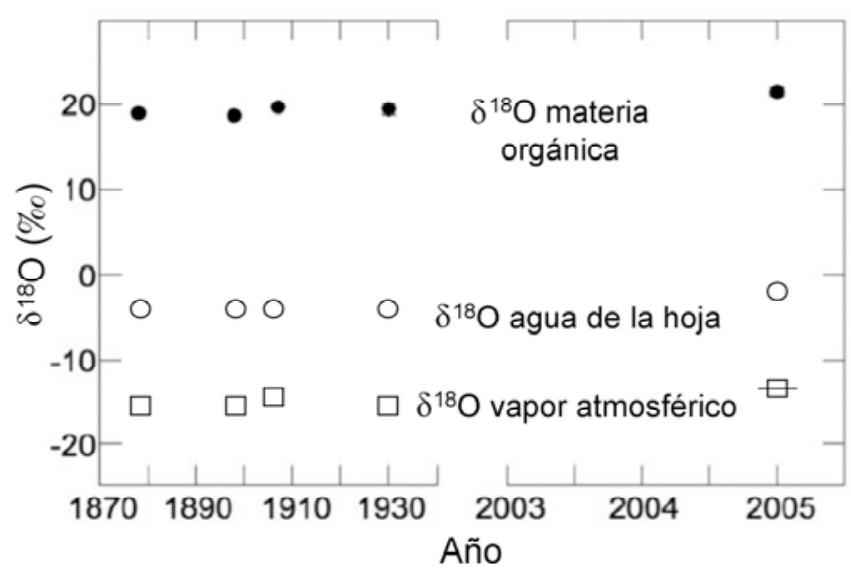

Figu ra 5. Estimación del $\delta^{18} \mathrm{O}$ del agua de la hoja de Tillandsia usneoides y del $\delta^{18} \mathrm{O}$ del vapor de agua de Miami, Florida. Los valores de $\delta^{18} \mathrm{O}$ del agua de la hoja y del vapor atmosférico fueron calculados a partir del $\delta^{18} \mathrm{O}$ de la mat e ria orgánica de esta especie, usando material fresco recolectado en septiembre de 2005, y de especímenes de herbario recolectados entre 1878 y 1930. Los cálculos se realizarm estimando que la mate ria orgánica de esta especie llega a ser $24.5 \%$ más pobre en $\delta^{18} \mathrm{O}$ que el agua de la hoja y que el vapor atmosférico estaría $10 \%$ más empobrecido en $\delta^{18} \mathrm{O}$ que el agua de la hoja. La línea hori zontal en el $\delta^{18} \mathrm{O}$ del vapor de agua para 2005 se calculó usando un promedio de 30 años de temperatura media de Miami $\left(23.3^{\circ} \mathrm{C}\right)$, al considerar que el vapor de agua está en equilibrio con el agua dulce de valor $\delta^{18} \mathrm{O}=-3.5 \%$ o (adaptado de Helliker y Gri ffiths, 2007).

abarcar escalas de tiempo desde el presente hasta miles de años atrás con las reconstrucciones climáticas y como marcadores de cambios en los ciclos hidrológicos y de carbono. Además, la gran diversidad morfológica y fisiológica de las plantas augura un aumento en las investigaciones con los isótopos estables del hidrógeno y oxígeno en los próximos años, especialmente en países megadiversos como México, donde las interrelaciones entre la gran diversidad de formas de vida, y entre éstas y su ambiente, pueden ser inspeccionadas con mayor precisión con la metodología de los isótopos estables.

\section{Agradecimientos}

A Howard Griffiths por discusiones acerca de los usos de los isótopos estables en diferentes disciplinas de la biología. Al Dr. Oscar Briones y un revisor anónimo por sus acertados comentarios en una ve rsión previa del manuscrito. Al Fondo Sectorial CONAFOR-CONACYT 2003-C0309765.

\section{Literatura citada}

Allison G.B., Gat J.R. y Leaney F.W.J. 1985. The relationship between deuterium and $\mathrm{O}^{18}$ delta values in leaf water. Chemical Geology 58:145-156.

Andrade J.L. 2003. Dew deposition on epiphytic bromeliad leaves: an important event in a Mexican tropical dry deciduous forest. Journal of Tropical Ecology 19:479-488.

Andrade J.L. 2005. Fisiología ecológica de árboles tropicales: avances y perspectivas. Revista Chapingo. Series Ciencias Forestales y del Ambiente 11:83-91.

Andrade J.L., Meinzer F.C., Goldstein G. y Schnitzer S.A. 2005. Water uptake and transport in lianas and co-occurring trees of a seasonally dry tropical forest. Trees - Structure and Function 19:282-289.

Aucour A.M., Hillaire-Marcel C. y Bonnefille R. 1996. Oxygen isotopes in cellulose from modern and quaternary intertropical peatbogs: implications for paleohydrology. Chemical Geology 129:341-359.

Baertschi P. 1976. Absolute ${ }^{18} \mathrm{O}$ content for standard mean ocean water. Earth and Planetary Science Letters 31:341-344.

Barbour M.M., Cernusak L.A. y Farquhar G.D. 2005. Factors affecting the oxygen isotope ratio of plant organic material. En: Flanagan L.B., Ehleringer J.R. y Pataki D.E. Eds. Stable Isotopes and Biosphere-Atmosphere Interactions: Processes and Biological Controls, pp. 9-28, Elsevier Academic Press, Amsterdam.

Barbour M.M., Fischer R.A., Sayre K.D. y Farquhar G.D. 2000a. Oxygen isotope ratio of leaf and grain material correlates with stomatal conductance and grain yield in irrigated wheat. Australian Journal of Plant Physiology 27:625-637.

Barbour M.M., Schurr U., Henry B.K., Wong S.C. y Farquhar G.D. 2000b. Variation in the oxygen isotope ratio of phloem sap sucrose from castor bean. Evidence in support of the Peclet effect. Plant Physiology 123:671-679.

Bariac T., Rambal S., Jusserand C. y Berger A. 1989. Evaluating water fluxes of field-grown alfalfa from diurnal observations of natural isotope concentrations, energy budget and ecophysiological parameters. Agricultural and Forest Meteorology 48:263-283.

Barnes B., Farquhar G. y Gan K. 2004. Modelling the isotope enrichment of leaf water. Journal of Mathematical Biology 48:672-702.

Beerling D.J. 1999. The influence of vegetation activity on the Dole effect and its implications for changes in biospheric productivity in the mid-Holocene. Proceedings of the Royal Society of London (Series B) 266:627-632.

Bender M., Sowers T. y Labeyrie L. 1994. The Dole effect and its variations during the last 130,000 years as measured in the Vostok ice core. Global Biogeochemical Cycles 8:363-376.

Bowen G.J. y Wilkinson B. 2002. Spatial distribution of $\delta^{18} \mathrm{O}$ in meteoric precipitation. Geology 30:315-318.

Burk R.L. y Stuiver M. 1981. Oxygen isotope ratios in trees reflect mean annual temperature and humidity. Science 211:1417-1419.

Cernusak L.A., Arthur D.J., Pate J.S. y Farquhar G.D. 2003. Water relations link carbon and oxygen isotope discrimination to phloem sap sugar concentration in Eucalyptus globulus. Plant Physiology 131:1544-1554.

Cernusak L.A., Farquhar G.D. y Pate J.S. 2005. Environmental and physiological controls over oxygen and carbon isotope composition of Tasmanian blue gum, Eucalyptus globulus. Tree Physiology 25:129-146.

Cooper L.W. y DeNiro M.J. 1989. Covariance of oxygen and hydrogen isotopic compositions in plant water - species effects. Ecology 70:1619-1628. 
Craig H. y Gordon L.I. 1965. Deuterium and oxygen-18 variations in the ocean and the marine atmosphere. En: Tongiorgi E. Ed. Proceedings of a Conference on Stable Isotopes in Oceanographic Studies and Paleotemperatures, pp. 9-130, Lischi and Figli, Pisa.

Danis P.A., Masson-Delmotte V., Stievenard M., Guillemim M.T., Daux V., Naveau P. y von Grafenstein U. 2006. Reconstruction of past precipitation beta $\mathrm{O}-18$ using tree-ring cellulose delta O-18 and delta C-13: A calibration study near Lac d'Annecy, France. Earth and Planetary Science Letters 243:439-448.

Dansgaard W. 1964. Stable isotopes in precipitation. Tellus Series B-Chemical and Physical Meteorology 16:436-468.

Dawson T.E., Mambelli S., Plamboeck A.H., Templer P.H. y Tu K.P. 2002. Stable isotopes in plant ecology. Annual Review of Ecology and Systematics 33:507-559.

DeNiro M.J. y Epstein S. 1981. Isotopic composition of cellulose from aquatic organisms. Geochimica et Cosmochimica Acta 45:1885-1894.

Dole M. 1935. Relative atomic weight in oxygen in water and in air. Journal of the American Chemical Society 57:2731-2731.

Ehleringer J.R. y Osmond C.B. 1989. Stable isotopes. En: Pearcy R.W., Ehleringer J.R., Mooney H.A. y Rundel P.W. Eds. Plant Physiological Ecology: Field Methods and Instrumentation, pp. 281-300, Chapman \& Hall, Londres.

Ehleringer J.R., Phillips S.L., Schuster W.S.F. y Sandquist D.R. 1991. Differential utilization of summer rains by desert plants. Oecologia 88:430-434.

Epstein S., Thompson P. y Yapp C.J. 1977. Oxygen and hydrogen isotopic ratios in plant cellulose. Science 198:1209-1215.

Farquhar G.D., Barbour M.M. y Henry B.K. 1998. Interpretation of oxygen isotope composition of leaf material. En: Griffiths H. Ed. Stable Isotopes: Integration of Biological and Geochemical Processes, pp. 27-6, BIOS Scientific Publishers, Oxford.

Farquhar G.D. y Cernusak L.A. 2005. On the isotopic composition of leaf water in the non-steady state. Functional Plant Biology 32:293-303.

Farquhar G.D. y Gan K.S. 2003. On the progressive enrichment of the oxygen isotopic composition of water along a leaf. Plant Cell and Environment 26:801-819.

Farquhar G.D. y Lloyd J. 1993. Carbon and oxygen isotope effects in the exchange of carbon dioxide between terrestrial plants and atmosphere. En: Ehleringer J.R., A.E. H. y Farquhar G.D. Eds. Stable Isotopes and Plant Carbon-Water Relations, pp. 47-70, Academic Press, San Diego.

Farquhar G.D., O'Leary M.H. y Berry J.A. 1982. On the relationship between carbon isotope discrimination and the intercellular carbon dioxide concentration in leaves. Australian Journal of Plant Physiology 21:221-234.

Farris F. y Strain B.R. 1978. Effects of water-stress on leaf $\left(\mathrm{H}_{2} \mathrm{O}\right)$ $\mathrm{O}^{18}$ enrichment. Radiation and Environmental Biophysics 15:167-202.

Feild T.S. y Dawson T.E. 1998. Water sources used by Didymopanax pittieri at different life stages in a tropical cloud forest. Ecology 79:1448-1452.

Flanagan L.B., Comstock J.P. y Ehleringer J.R. 1991. Comparison of modeled and observed environmental influences on the stable oxygen and hydrogen isotope composition of leaf water in Phaseolus vulgaris L. Plant Physiology 96:588-596.

Gat J.R. 1996. Oxygen and hydrogen isotopes in the hydrologic cycle. Annual Review of Earth and Planetary Sciences 24:225-
262.

Gat J.R. 1998. Stable isotopes, the hydrological cycle and atmospheric processes. En: Griffiths H. Ed. Stable Isotopes: Integration of Biological and Geochemical Processes, pp. $397-$ 407, BIOS Scientific Publishers, Oxford.

Gat J.R., Mook W.G. y Meijer H.A.J. 2000. Atmospheric water. En: Mook W.G. Ed. Environmental Isotopes in the Hydrological Cycle, pp. 1-111, UNESCO/IAEA, Groningen.

Goldstein G., Meinzer F.C. y Andrade J.L. 2002. El flujo de agua en los árboles del dosel: mecanismos y patrones. En: Guariguata M.R y Kattan G.H. Eds. Ecología y Conservación de Bosques Neotropicales, pp. 251-270, Libro Universitario Regional, Cartago, Costa Rica.

Gray J. y Thompson P. 1976. Climatic information from ${ }^{18} \mathrm{O} /{ }^{16} \mathrm{O}$ ratios of cellulose in tree rings. Nature 262:481-482.

Hagemann R., Nief G. y Roth E. 1970. Absolute isotopic scale for deuterium analysis of natural waters. Absolute $\mathrm{D} / \mathrm{H}$ ratio for SMOW. Tellus Series B-Chemical and Physical Meteorology 22:712-715.

Harwood K.G., Gillon J.S., Griffiths H. y Broadmeadow M.S.J. 1998. Diurnal variation of delta $\left(\mathrm{CO}_{2}\right)-\mathrm{C}^{13}$, delta $(\mathrm{COO})-\mathrm{O}^{18}-\mathrm{O}^{16}$ and evaporative site enrichment of delta $\left(\mathrm{H}_{2} \mathrm{O}\right)-\mathrm{O}^{18}$ in Piper aduncum under field conditions in Trinidad. Plant Cell and Environment 21:269-283.

Harwood K.G., Gillon J.S., Roberts A. y Griffiths H. 1999. Determinants of isotopic coupling of $\mathrm{CO}_{2}$ and water vapour within a Quercus petraea forest canopy. Oecologia 119:109119.

Helliker B.R. y Ehleringer J.R. 2000. Establishing a grassland signature in veins: $\mathrm{O}^{18}$ in the leaf water of $\mathrm{C}_{3}$ and $\mathrm{C}_{4}$ grasses. Proceedings of the National Academy of Sciences of the United States of America 97:7894-7898.

Helliker B.R. y Ehleringer J.R. 2002a. Grass blades as tree rings: environmentally induced changes in the oxygen isotope ratio of cellulose along the length of grass blades. New Phytologist 155:417-424.

Helliker B.R. y Ehleringer J.R. 2002b. Differential $\mathrm{O}^{18}$ enrichment of leaf cellulose in $\mathrm{C}_{3}$ versus $\mathrm{C}_{4}$ grasses. Functional Plant Biology 29:435-442.

Helliker B.R. y Griffiths H. 2007. Towards a plant-based proxy for the ${ }^{18} \mathrm{O}$ atmospheric water vapor. Global Change Biology 13:723-733.

Helliker B.R., Roden J.S., Cook C. y Ehleringer J.R. 2002. A rapid and precise method for sampling and determining the ox yen isotope ratio of atmospheric water vapor. Rapid Communications in Mass Spectrometry 16:929-932.

Henderson-Sellers A., McGuffie K. y Zhang H. 2002. Stable isotopes as validation tools for global climate model predictions of the impact of Amazonian deforestation. Journal of Climate 15:2664-2677.

Hoefs J. 2004. Stable Isotope Geochemistry. 5a. ed. Springer, Berlín.

Hoffmann G., Cuntz M., Weber C., Ciais P., Friedlingstein P., Heimann M., Jouzel J., Kaduk J., Maier-Reimer E., Seibt U. y Six K. 2004. A model of the Earth's Dole effect. Global Biogeochemical Cycles 18:1-15.

IAEA/WMO [International Atomic Energy Agency/World Meteorological Organization]. 2004. Global Network of Isotopes in Precipitation.

<isohis.iaea.org/gnip.asp> 
Jackson P.C., Cavelier J., Goldstein G., Meinzer F.C. y Holbrook N.M. 1995. Partitioning of water resources among plants of a lowland tropical forest. Oecologia 101:197-203.

Jackson P.C., Meinzer F.C., Bustamante M., Goldstein G., Franco A., Rundel P.W., Caldas L., Igler E. y Causin F. 1999. Partitioning of soil water among tree species in a Brazilian Cerrado ecosystem. Tree Physiology 19:717-724.

Majoube M. 1971. Oxygen-18 and deuterium fractionation between water and steam. Journal de Chimie Physique et de Physico-Chimie Biologique 68:1423-1436.

Malaize B., Paillard D., Jouzel J. y Raynaud D. 1999. The Dole effect over the last two glacial-interglacial cycles. Journal of Geophysical Research-Atmospheres 104:14199-14208.

Martinelli L.A., Gat J.R., De Camargo P.B., Lara L.L. y Ometto J.P.H. 2004. The Piracicaba river basin: isotope hydrology of a tropical river basin under anthropogenic stress. Isotopes in Environmental and Health Studies 40:45-56.

Meinzer F.C., Andrade J.L., Goldstein G., Holbrook N.M., Cavelier J. y Wright S.J. 1999. Partitioning of soil water among canopy trees in a seasonally dry tropical forest. Oecologia 121:293-301.

Meinzer F.C., Clearwater M.J. y Goldstein G. 2001. Water transport in trees: curnent perspectives, new insights and some controversies. Environmental and Experimental Botany 45:239-262.

Moreira M.Z., Scholz F.G., Bucci S.J., Sternberg L.S., Goldstein G., Meinzer F.C. y Franco A.C. 2003. Hydraulic lift in a neotropical savanna. Functional Ecology 17:573-581.

Moreira M., Sternberg L.D., Martinelli L.A., Victoria R., Barbosa E., Bonates L. y Nepstad D.C. 1997. Contribution of transpiration to forest ambient vapour based on isotopic measurements. Global Change Biology 3:439-450.

Nobel P.S. 1991. Psychochemical and Environmental Plant Physiology. Academic Press, San Diego.

Pendall E., Williams D.G. y Leavitt S.W. 2005. Comparison of measured and modeled variations in pinon pine leaf water isotopic enrichment across a summer moisture gradient. Oecologia 145:605-618.

Phillips S.L. y Ehleringer J.R. 1995. Limited uptake of summer precipitation by bigtooth maple (Acer grandidentatum Nutt) and Gambel's oak (Quercus gambelii Nutt). Trees 9:214-219.

Pollard A.M. 1998. Archeological reconstruction using stable isotopes. En: Griffiths H. Ed. Stable Isotopes: Integration of Biological and Geochemical Processes, pp. 285-301, BIOS Scientific Publisher, Oxford.

Rozanski K., Araguas-Araguas L. y Gonfiantini R. 1993. Isotopic patterns in modern global precipitation. En: Swart P.K., Lohman J.A., McKenzie J.A. y Savin S. Eds. Climate Change in Continental Isotopic Records, pp. 1-36, Geophysical Monographies, Washington, D.C.

Santiago L.S., Silvera K., Andrade J.L. y Dawson T.E. 2005. El uso de isótopos estables en biología tropical. Interciencia 30:28-35.
Seibt U., Wingate L., Berry J.A. y Lloyd J. 2006. Non-steady state effects in diurnal ${ }^{18} \mathrm{O}$ discrimination by Picea sitchensis branches in the field. Plant, Cell and Environment 29:928-939.

Siegenthaler U. y Oesch ger H. 1980. Correlation of ${ }^{18} \mathrm{O}$ in precipitation with temperature and altitude. Nature 285:314-317.

Sternberg L.d.S.L. 1989. Oxygen and hydrogen isotope ratios in plant cellulose: mechanisms and applications. En: Rundel P.W., Ehleringer J.R. y Nagy K.A. Eds. Stable Isotopes in Ecological Research, pp.124-141, Springer Verlag, Nueva York.

Stemberg L.d.S.L., Anderson W.T. y Morrison K.M. 2003. Separating soil and leaf water ${ }^{18} \mathrm{O}$ isotopic signals in plant stem cellulose. Geochimica et Cosmochimica Acta 67:2561-2566.

Sternberg L.d.S.L. y DeNiro M.J.D. 1983. Biochemical implications of the isotopic equilibrium fractionation factor between oxygen atoms of acetone and water. Geochimica et Cosmochimica Acta 47:2271-2274.

Sternberg L.d.S.L., DeNiro M.J.D. y Savidge R.A. 1986. Oxygen isotope exchange between metabolites and water during biochemical reactions leading to cellulose synthesis. Plant Physiology 82:423-427.

Sternberg L.d.S.L., Moreira M.Z. y Nepstad D.C. 2002. Uptake of water by lateral roots of small trees in an Amazonian tropical forest. Plant and Soil 238:151-158.

Sternberg L.d.S.L. y Swart P.K. 1987. Utilization of fresh water and ocean water by coastal plants of southern Florida. Ecology 68:1898-1905.

Tissue D.T., Yakir D. y Nobel P.S. 1991. Diel water-movement between parenchyma and chlorenchyma of two desert CAM plants under dry and wet conditions. Plant Cell and Environment 14:407-413.

Wang X.F. y Yakir D. 1995. Temporal and spatial variations in the oxygen-18 content of leaf water in different plant species. Plant Cell and Environment 18:1377-1385.

White J.W.C., Cook E.R., Lawrence J.R. y Broecker W.S. 1985. The $\mathrm{D} / \mathrm{H}$ ratios of sap in trees: Implications for water sources and tree ring D/H ratios. Geochimica et Cosmochimica Acta 49:237-246.

Yakir D. 1998. Oxygen-18 of leaf water: a crossroad for plantassociated isotopic signals. En: Griffiths H. Ed. Stable Isotopes: Integration of Biological Ecological and Geochemical Processes, pp. 147-168, BIOS Scientific Publishers Limited, Oxford.

Yakir D. , Berry J. A., Giles L. y Osmond C.B. 1994. Isotopic hete rogeneity of water in transpiring leaves - Identification of the component that controls the delta- $\mathrm{O}^{18}$ of atmospheric $\mathrm{O}^{2}$ and $\mathrm{CO}_{2}$. Plant Cell and Environment 17:73-80.

Yakir D. y DeNiro M.J. 1990. Oxygen and hydrogen isotope fractionation during cellulose metabolism in Lemna gibba L. Plant Physiology 93:325-332.

Yapp C.J. y Epstein S. 1982. Climatic significance of the hydrogen isotope ratios in tree cellulose. Nature 297:636-639.

Recibido: 16 de febrero de 2007

Versión corregida: 16 de abril de 2007

Aceptado: 17 de abril de 2007 